\title{
Oscillations of a suspended slinky
}

\author{
Jörg Pretz \\ Institut für Kernphysik, Forschungszentrum Jülich, 52425 Jülich, Germany \\ III. Physikalisches Institut B, RWTH Aachen University, 52056 Aachen, Germany \\ JARA-FAME, Forschungszentrum Jülich und RWTH Aachen University \\ E-mail: pretz@physik.rwth-aachen.de
}

May 2020

\begin{abstract}
This paper discusses the oscillations of a spring (slinky) under its own weight. A discrete model, describing the slinky by $N$ springs and $N$ masses, is introduced and compared to a continuous treatment. One interesting result is that the upper part of the slinky performs a triangular oscillation whereas the bottom part performs an almost harmonic oscillation if the slinky starts with "natural" initial conditions, where the spring is just pulled further down from its rest position under gravity and then released.

It is also shown that the period of the oscillation is simply given by $T=\sqrt{32 L / g}$, where $L$ is the length of the slinky under its own weight and $g$ the acceleration of gravity independent of the other properties of the spring.
\end{abstract}

Keywords: slinky, harmonic oscillations

Submitted to: Eur. J. Phys.

\section{Introduction}

A slinky, invented in the 1940 by Richard James, is in the context of this paper a spring that is oscillates under its own weight without any additional mass attached to it with a quality factor high enough to observe the oscillations. There are many articles on a falling slinky [1, 2, 3] and the suspended slinky [4, 5]. This article studies interesting aspects of a suspended slinky. Equations of motion are derived and solved for the discrete case where the slinky is described by $N$ masses and springs (section 2). Section 3 treats the continuous case. Section 4 compares the results obtained analytically to experimental results.

\section{Discrete case}

The slinky is modeled by $N$ identical massless springs with spring constant $d$ and mass $m$ as shown in Figure 1. A given mass experiences forces from the two neighboring springs leading to the following equation of motion

$$
m \ddot{x}_{j}=-d\left(x_{j}-x_{j-1}\right)-d\left(x_{j}-x_{j+1}\right),
$$


where the $x_{j}$ denotes the excursion from the rest position of the mass. An exception is the first and last spring. In this case the equations of motion read

$$
\begin{aligned}
& m \ddot{x}_{1}=-d x_{1}-d\left(x_{1}-x_{2}\right), \\
& m \ddot{x}_{N}=-d\left(x_{N}-x_{N-1}\right) .
\end{aligned}
$$

This results in the following system of coupled differential equations

$$
\ddot{\boldsymbol{x}}+\omega_{0}^{2} B \boldsymbol{x}=0,
$$

with

$$
B=\left(\begin{array}{rrrrrr}
2 & -1 & 0 & 0 & \ldots & 0 \\
-1 & 2 & -1 & 0 & \ldots & 0 \\
0 & -1 & 2 & -1 & \ldots & 0 \\
\vdots & & & \ddots & & \vdots \\
0 & \ldots & 0 & -1 & 2 & -1 \\
0 & \ldots & 0 & 0 & -1 & 1
\end{array}\right)
$$

and

$$
\omega_{0}^{2}=d / m
$$

In contrast to the corresponding matrix of a falling slinky [3], there is a 2 in the upper left corner instead of a 1.

A solution with initial condition $\dot{\boldsymbol{x}}(0)=0$ is:

$$
\boldsymbol{x}(t)=\boldsymbol{a} \cos (\omega t) .
$$

Inserting equation 7 into equation 4 leads to the eigenvalue problem

$$
-\omega^{2} \boldsymbol{a}+\omega_{0}^{2} B \boldsymbol{a}=0 .
$$

The general solution of equation 4 is thus

$$
\boldsymbol{x}(t)=\sum_{j=0}^{N} c_{j} \boldsymbol{a}_{j} \cos \left(j \omega_{j} t\right),
$$

where $\omega_{j}=\sqrt{\left|\omega_{j}^{2}\right|}$ are the the square roots of the eigenvalues and $\boldsymbol{a}_{j}$ the corresponding eigenvectors. For a given initial condition $\boldsymbol{x}(0)=\boldsymbol{x}_{0}$, the vector $\boldsymbol{c}$ is determined by inverting

$$
\boldsymbol{x}_{0}=A \boldsymbol{c}
$$

where $A$ is the matrix with the eigenvectors as columns.

We assume the springs to be massless and of zero length when not exposed to a force. Then the rest position of the first (top) mass is given by

$$
\left(\boldsymbol{x}_{\text {rest }}\right)_{1}=N \frac{m g}{d} \text {. }
$$

The first spring is stretched by all $N$ masses. The second spring is accordingly stretched by $N-1$ masses. This leads to

$$
\boldsymbol{x}_{\mathrm{rest}}=\frac{m g}{d}(N, N+(N-1), N+(N-1)+(N-2), \ldots) \text {. }
$$




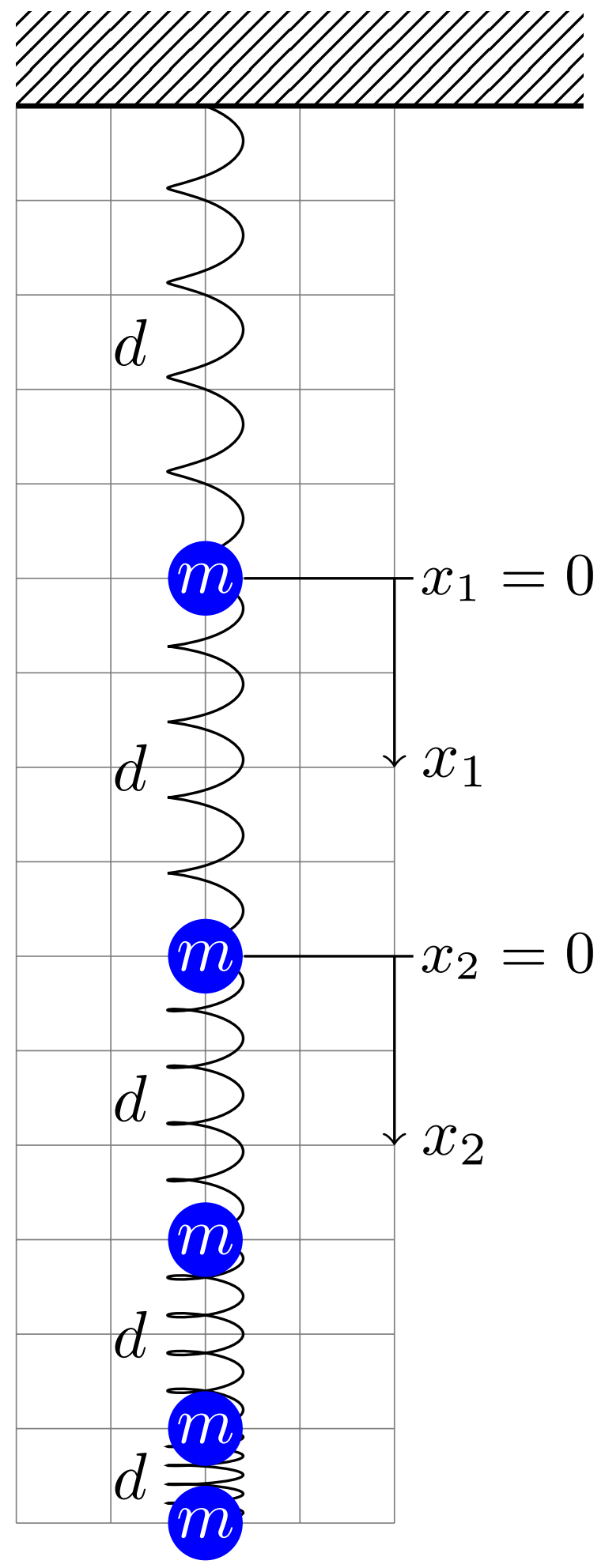

Figure 1. Slinky modeled as $N$ masses and $N$ springs. The picture shows the rest position under its own weight. 
Table 1. Parameters for oscillations shown in Figure 2.

\begin{tabular}{|c|c|c|}
\hline parameter & value & meaning \\
\hline$N$ & 10 & number of masses \\
\hline$X_{0} / \mathrm{m}$ & 0.1 & deviation from rest position for bottom mass \\
\hline$D / \mathrm{kg} \mathrm{s}^{-2}$ & 0.15 & spring constant of slinky \\
\hline$d=N D / \mathrm{kg} \mathrm{s}^{-2}$ & 1.5 & $\begin{array}{l}\text { corresponding spring constant of single spring } \\
\text { between two masses in Fig. } 1 .\end{array}$ \\
\hline$M / g$ & 30 & mass of slinky \\
\hline
\end{tabular}

The position of the $N$-th mass is thus

$$
\left(\boldsymbol{x}_{\text {rest }}\right)_{N}=\frac{m g}{d} \frac{N(N+1)}{2}=L \text {. }
$$

To study oscillations we just pull the slinky further down from its rest position. The initial condition (deviation from $\boldsymbol{x}_{\text {rest }}$ ) is given by

$$
\boldsymbol{x}_{0}=X_{0} \frac{2}{N(N+1)} \frac{d}{m g} \boldsymbol{x}_{\text {rest }} .
$$

For the first and last mass from one finds:

$$
\left(\boldsymbol{x}_{0}\right)_{1}=\frac{2}{N+1} X_{0}, \quad\left(\boldsymbol{x}_{0}\right)_{N}=X_{0} \text {. }
$$

Now that the initial conditions are fixed one can calculate the oscillations using equation 9. Figure 2 shows the results for the parameters given in table 11. The amplitudes are the larger the lower the position along the slinky. At the top, the oscillations have a triangular shape whereas at the bottom the oscillation is more sinusoidal. In the next section we try to understand this behavior by going to a continuous distribution of the mass over the slinky.

\section{Continuous case}

If $N \rightarrow \infty$ and $m \rightarrow 0$ such that $N m=M$ remains constant, one reaches a continuous mass distribution. This system can best be described by a dimensionless variable $n$ which is defined by the turn number of the spring divided by the total number of turns, i.e. $0 \leq n \leq 1[5]$. The position $x$ as a function of $n$ along the slinky under gravity can be derived from the following consideration. Under gravity the stretching $x(n+d n)-x(n)$ of the slinky is proportional to the remaining mass below the position $n$. Since this mass is proportional to $(1-n)$, one finds:

$$
x(n+\mathrm{d} n)-x(n) \propto(1-n) \mathrm{d} n .
$$

This leads to

$$
x(n)=\left(2 n-n^{2}\right) L,
$$

normalized such that the total length is $x(1)=L$. Inverting equation 16 leads to

$$
n(x)=1-\sqrt{1-\left(\frac{x}{L}\right)} \text {. }
$$




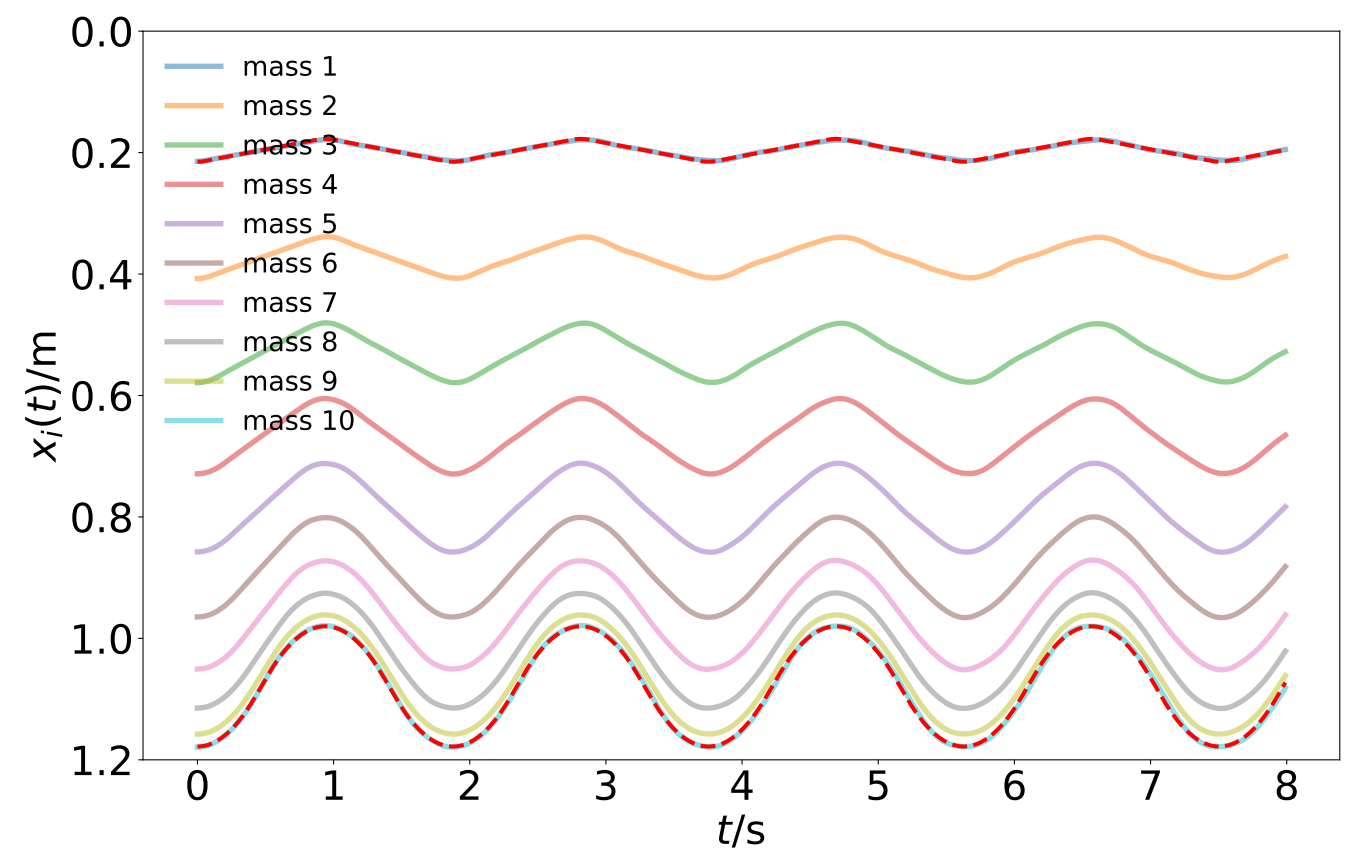

Figure 2. Oscillations as a function of time $t$ for the different masses.

Appendix A shows in detail that, using $n$ instead of the vertical position $x$ as a coordinate, the system can be described by the following wave equation [5, 6]

$$
\frac{\partial^{2} s(n, t)}{\partial t^{2}}-\frac{g}{2 L} \frac{\partial^{2} s(n, t)}{\partial n^{2}}=0,
$$

where a wave is propagating with constant velocity $v=\sqrt{\frac{g}{2 L}}$. This is not the case if the coordinate $x$ is used instead of $n . s(n, t)$ is the deviation of the slinky from its rest position as a function of the relative turn number $n$ and time $t$.

If we pull the slinky at the bottom, according to equation 16 the initial conditions are given by

$$
s(n, 0)=\left(2 n-n^{2}\right) X_{0},
$$

where $X_{0}$ denotes the excursion of the bottom of the slinky from its rest position. Further initial and boundary conditions are

$$
\begin{aligned}
& \dot{s}(n, 0)=0, \\
& s(0, t)=0 \text { and } \\
& \frac{\mathrm{d} s(1, t)}{\mathrm{d} n}=0 .
\end{aligned}
$$

Equation 22 assures an anti-node at the open end $n=1$.

The solution is given by

$$
s(n, t)=\sum_{j=0}^{\infty} A_{j} \sin \left(k_{j} n\right) \cos \left(\omega_{j} t\right) .
$$


To fulfill condition equation 22 , we have

$$
k_{j}=(2 j+1) \frac{\pi}{2}, \quad j=0,1,2, \ldots
$$

Finally equation 18 leads to:

$$
\omega_{j}=\sqrt{\frac{g}{2 L}} k_{j} \text {. }
$$

The fundamental frequency is thus given by

$$
\omega_{0}=\sqrt{\frac{g}{2 L}} \frac{\pi}{2} .
$$

For the period length of the slinky one finds

$$
T=\sqrt{\frac{32 L}{g}},
$$

independent of the mass or other properties of the slinky. This result has been derived in [5] in a different context as a round trip time of a pulse.

The length $L$ under its own weight is easily derived from equation 13

$$
L=\lim _{N \rightarrow \infty, m \rightarrow 0} \frac{m g}{d} \frac{N(N+1)}{2}=\frac{M g}{2 D} .
$$

Note that $d$ is the spring constant of a single spring (see Fig. 1). The spring constant of the total spring is $D=d / N$. The dependence of the period duration $T$ on $M$ and $D$ is hidden in the length $L(M, D)$. $T$ can also be expressed as

$$
T=4 \sqrt{\frac{M}{D}} .
$$

For the parameters given in table 1 , one finds $\omega_{0}=3.49 \mathrm{~s}^{-1}$ which is very close to the solution found in the discrete case for $N=10, \omega_{0, N=10}=3.34 \mathrm{~s}^{-1}$. Figure 3 shows a comparison of the frequencies for various values of $N$ for the first three contributing frequencies.

The coefficients $A_{i}$ in equation 23 are found by a Fourier analysis. As shown in detail in Appendix B, in order to satisfy the initial condition equation 19, one finds

$$
A_{i} \propto \frac{32}{\pi^{3}(2 j+1)^{3}} X_{0}, \quad j=0,1,2,3, \ldots .
$$

The amplitude of the different frequencies is given by the factors $A_{j} \sin \left(k_{j} n\right)$ in equation 23. At small $n$, i.e. at the top of the spring, one finds

$$
A_{j} \sin \left(k_{j} n\right)=X_{0} \frac{32}{\pi^{3}(2 j+1)^{3}} k_{i} n=X_{0} \frac{16}{\pi^{2}(2 j+1)^{2}} n .
$$

The amplitudes have the ratios 1:9:25: . which corresponds to a triangular shape as observed in Figure 2. At the bottom end of the slinky, i.e. $n=1$ one has $\sin \left(k_{j} n\right)=1$ resulting in

$$
\begin{aligned}
A_{j} \sin \left(k_{j} n\right) & =X_{0} \frac{32}{\pi^{3}} \approx 1.032 X_{0} \text { for } j=0 \\
& =X_{0} \frac{32}{27 \pi^{3}} \approx 0.038 X_{0} \text { for } j=1 \\
& =X_{0} \frac{32}{125 \pi^{3}} \approx 0.0083 X_{0} \text { for } j=2,
\end{aligned}
$$




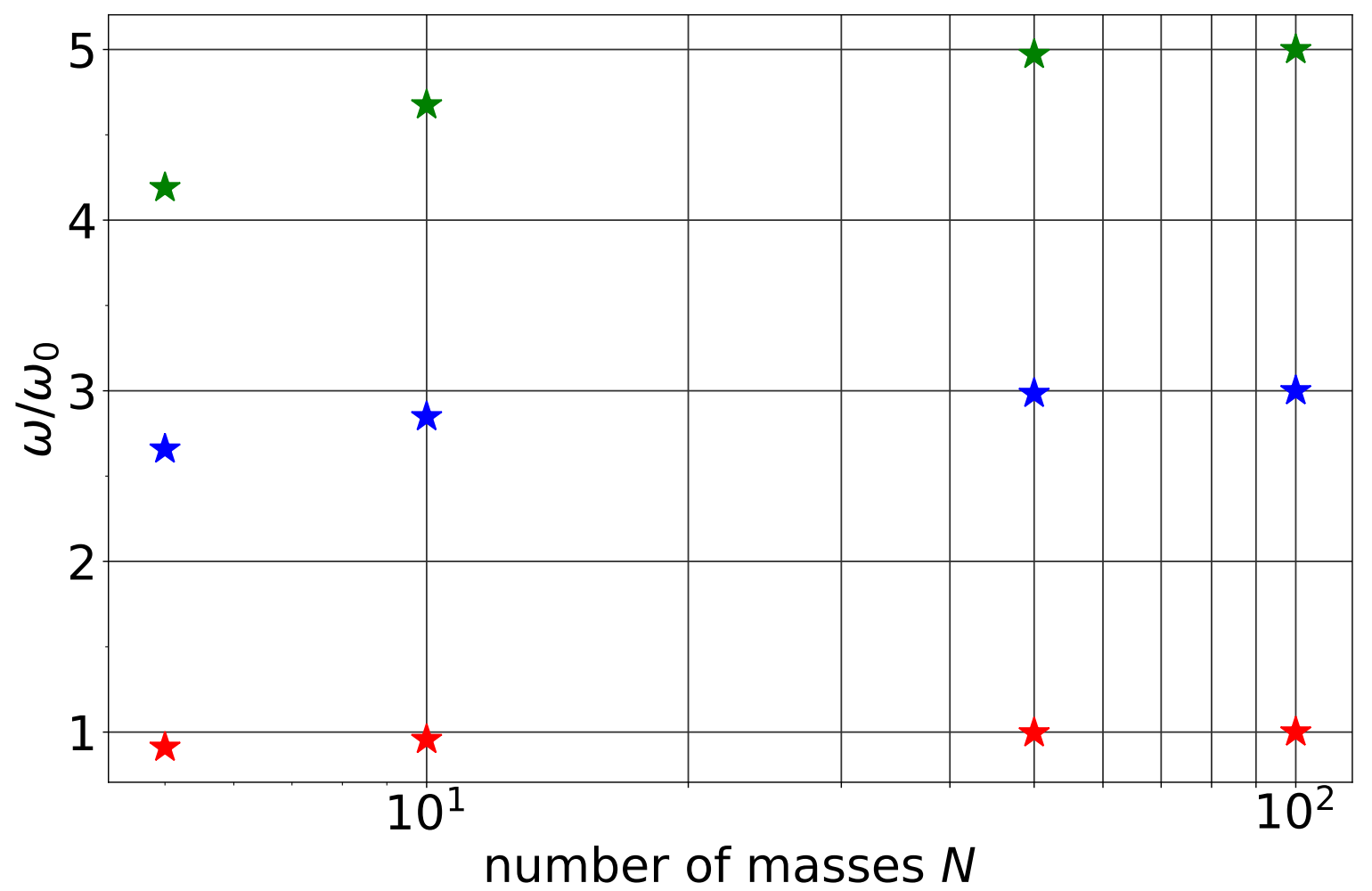

Figure 3. First three frequencies for $N=5,10,50,100$ divided by the fundamental frequency $\omega_{0}$ for the continuous case.

Table 2. Parameters of slinkies used in experiments.

\begin{tabular}{lll}
\hline & plastic & metal \\
\hline nb. of turns & 52 & 93 \\
mass $M / \mathrm{g}$ & 65 & 35 \\
length $L / \mathrm{m}$ under gravity & 2.40 & 0.32 \\
unstretched length $L_{0} / \mathrm{m}$ & 0.07 & 0.04 \\
\hline
\end{tabular}

which almost corresponds to a pure sine wave. But even in the limit $N \rightarrow \infty$ the bottom mass oscillation is not a purely harmonic.

In Fig. 2 the dotted lines for the top and bottom mass show the solution (at $n=0.1$ and $n=1$ respectively) from the wave equation which agrees perfectly with the discrete solution with $N=10$.

\section{Comparison to experiment}

Two slinkies (see Figure 5 and Table 2) were used to confirm equation 26 and to verify the motion of the top and bottom mass.

Figure 4 shows a comparison of the measured and calculated period duration. A 
Table 3. Results of the measurements with the two slinkies. $L_{0}$ is the length in the unstretched state. The numbers in parentheses indicate the uncertainties.

\begin{tabular}{lllll}
\hline length $L / \mathrm{m}$ & length $L_{0} / \mathrm{m}$ & $T_{\text {meas }} / \mathrm{s}$ & $T_{\text {theo }} / \mathrm{s}$ & \\
\hline $2.400(5)$ & $0.070(2)$ & $2.50(2)$ & $2.80(13)$ & plastic \\
$1.000(5)$ & $0.040(2)$ & $1.65(2)$ & $1.81(13)$ & plastic \\
$0.320(5)$ & $0.040(2)$ & $1.01(2)$ & $1.02(13)$ & metal \\
$0.090(5)$ & $0.020(2)$ & $0.59(2)$ & $0.54(13)$ & metal \\
\hline
\end{tabular}

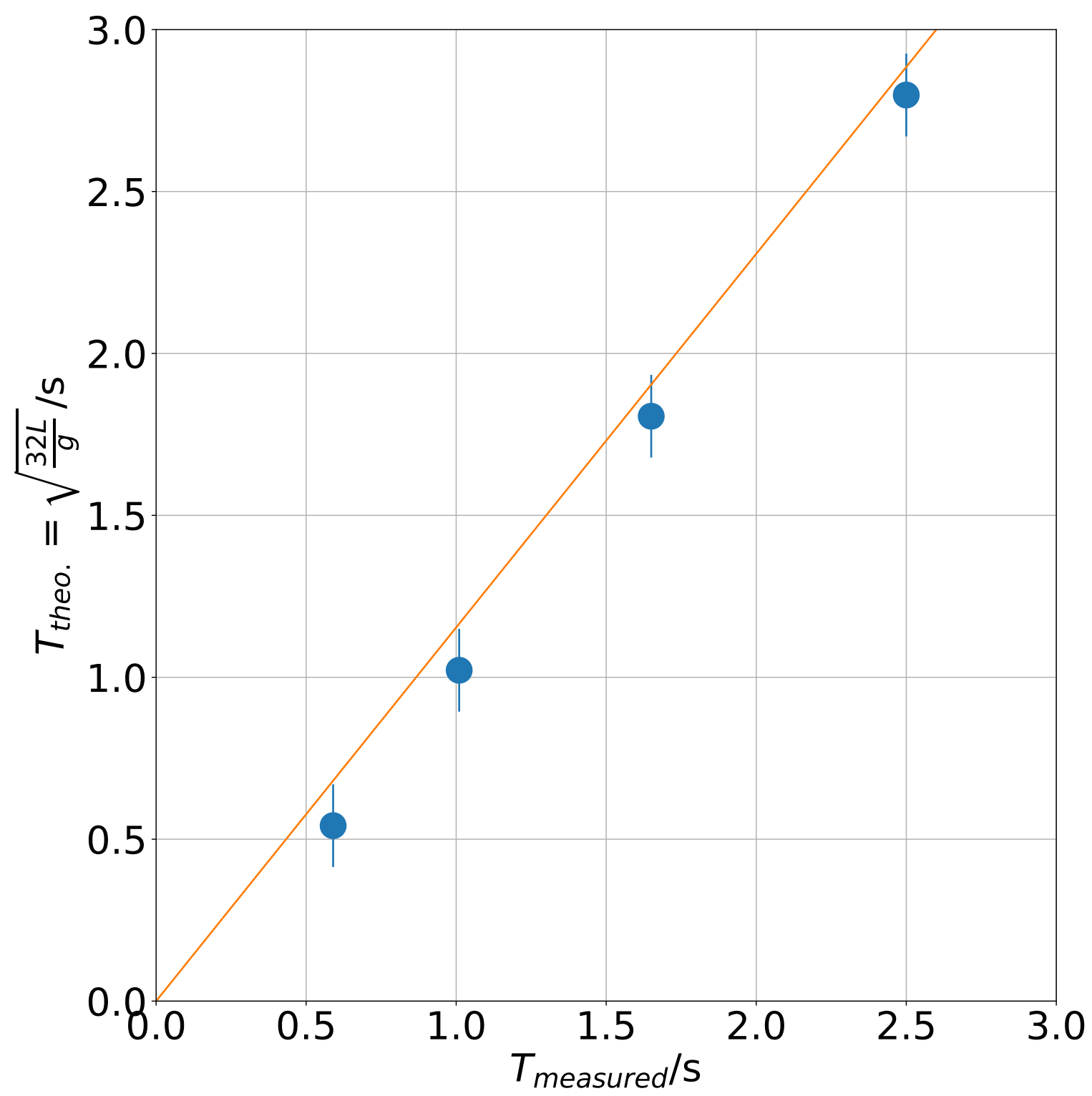

Figure 4. The calculated period length (equation 26) vs. the measured period length. 


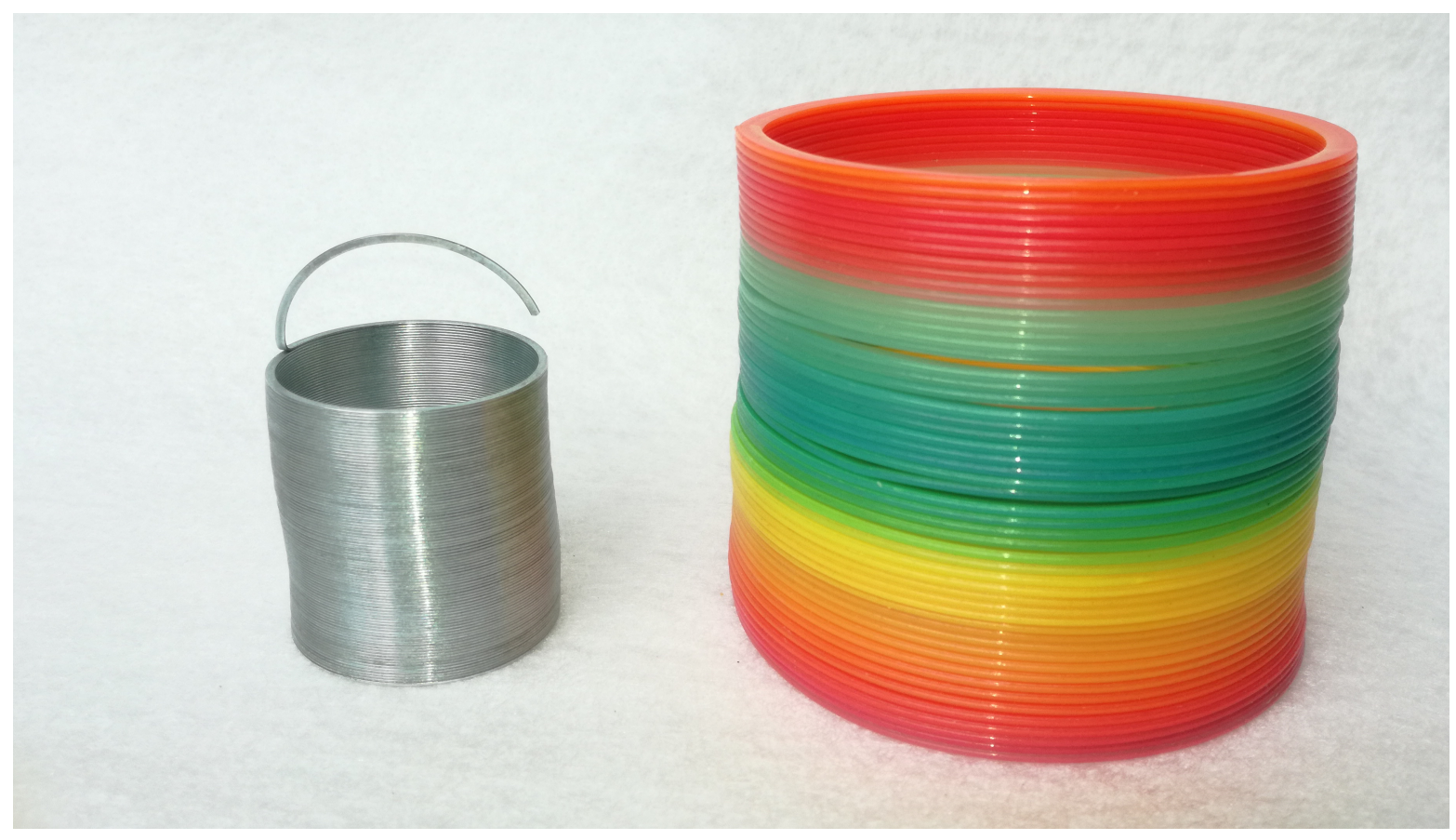

Figure 5. Photograph of the two slinkies used in the experiemnts.

reasonable agreement is found.

Figure 6 shows the measurement of an oscillating slinky. The top plots are the oscillations and frequencies of a point at the top of the slinky, the bottom plots are the corresponding plots for the bottom end of the slinky. Qualitatively one observes the behavior in Figure 2. The oscillation of the top point is more triangular shaped. It is worthwhile to mention that in these experiments it is important to pull the slinky from the bottom to have the correct initial conditions. If the slinky is for instance pulled at $n \approx 0.4$, the oscillations look very different. In principle this could be studied by choosing the corresponding initial conditions in equations 10 or 19 .

The points of Figure 6 were obtained using the open source opencv library [7] which allows easily to track a point in a video according to its color. Figure 7 shows the analysis of five video frames, where a point at the bottom of the slinky is tracked.

\section{Summary and conclusions}

Starting from discrete treatment with $N$ masses attached to $N$ springs the vertical motion of a suspended slinky was studied. A first observation of the discrete treatment is that the top part of the slinky performs triangular motions whereas the bottom part is more sinusoidal. Using a continuous model this could also be confirmed analytically. Measurements show a good agreement with the analytic results derived. 


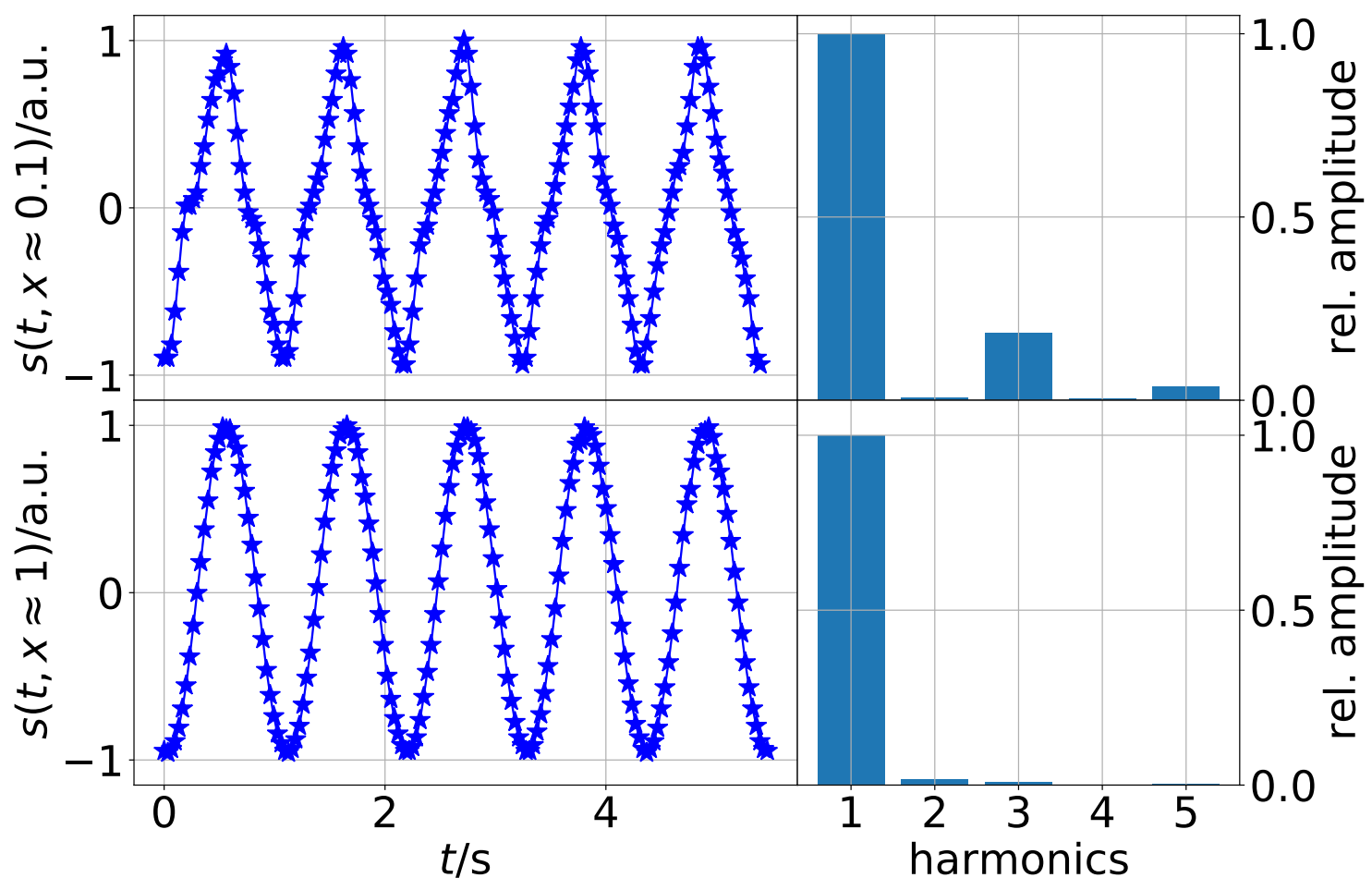

Figure 6. Left: Oscillation of top and bottom part of the slinky. Right: The corresponding frequency spectrum.

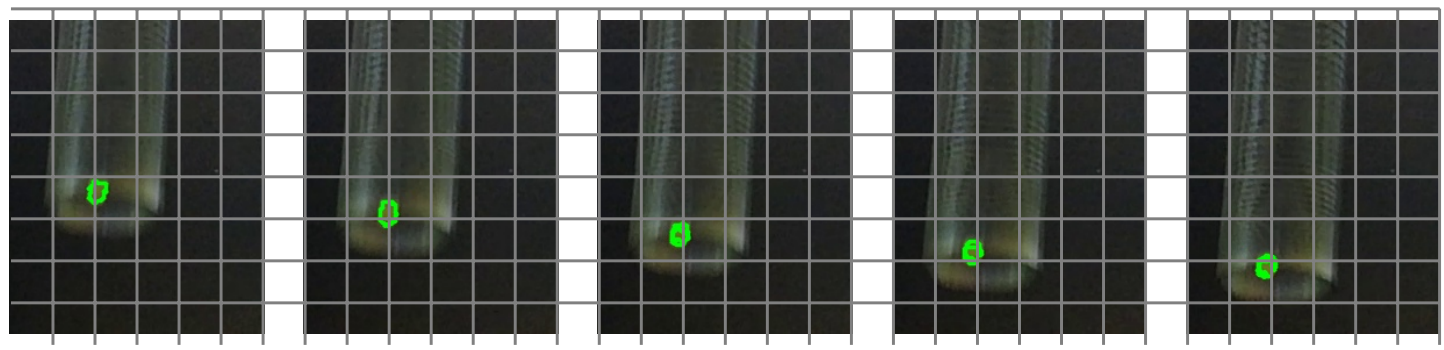

Figure 7. Analysis of five video frames using the opencv library to track a point at the bottom of the slinky [7]. The result of the tracking is indicated by the green areas. The images are taken $0.033 \mathrm{~s}$ apart. 


\section{Acknowledgments}

I would like to thank J. Barth for comments and suggestions on the paper. 


\section{Appendix A. Derivation of the wave equation}

The wave equation for a string subject to a force $F$ along the longitudinal direction reads

$$
\frac{\partial^{2} s(x, t)}{\partial t^{2}}-\frac{F}{\rho A} \frac{\partial^{2} s(x, t)}{\partial x^{2}}=0,
$$

where $\rho$ is the density and $A$ the cross-sectional area of the string.

The force at a given $n$ is given by

$$
F(n)=(1-n) M g
$$

where $M$ is the total mass of the slinky.

From equations 16 and A.2 we can express the force and the density $\rho$ as a function of the position $x$.

$$
\begin{aligned}
& F(x)=(1-n(x)) M g=\sqrt{1-\frac{x}{L}} M g, \\
& \rho(x)=\frac{\mathrm{d} n}{\mathrm{~d} x} \frac{M}{A}=\frac{1}{L} \frac{1}{2 \sqrt{1-\left(\frac{x}{L}\right)}} \frac{M}{A} .
\end{aligned}
$$

This leads to

$$
\frac{\partial^{2} s(x, t)}{\partial t^{2}}-2 g L\left(1-\frac{x}{L}\right) \frac{\partial^{2} s(x, t)}{\partial x^{2}}=0 .
$$

As in references [5, 6] it is easier to write the wave equation in terms of the turn number $n$

$$
\tilde{s}(n, t)=s(x(n), t) \frac{d x}{d n}=s(x(n), t) 2 L \sqrt{1-\left(\frac{x}{L}\right)}
$$

Using

$$
\frac{\partial}{\partial x}=\frac{\partial}{\partial n} \frac{d n}{d x}=\frac{\partial}{\partial n}\left(2 L \sqrt{1-\left(\frac{x}{L}\right)}\right)^{-1}
$$

and dropping the tilde on $s$, the wave equation becomes

$$
\frac{\partial^{2} s(n, t)}{\partial t^{2}}-\frac{g}{2 L} \frac{\partial^{2} s(n, t)}{\partial n^{2}}=0 .
$$

In terms of the relative turn number $n$ the velocity $\mathrm{d} n / \mathrm{d} t=\sqrt{\frac{g}{2 L}}$ is constant.

\section{Appendix B. Determination of coefficients $A_{i}$}

Equations 19 and 23 for $t=0$ read

$$
X_{0}\left(2 n-n^{2}\right)=\sum_{j=0}^{\infty} A_{j} \sin \left(k_{j} n\right)
$$

For $j=0$ the sine term performs only one quarter of an oscillation between $n=0$ and $n=1$. Therefore the following integral is only evaluated over a quarter period length 
up to $\lambda / 4$. To get the correct normalization the result has to be multiplied by 4 . This results in the following expression for the Fourier coefficients $A_{i}$ :

$$
A_{i}=4 \int_{0}^{\lambda / 4} \sin (k n)\left(2 n-n^{2}\right) X_{0} \mathrm{~d} n \frac{k_{0}}{\pi}=\frac{32}{\pi^{3}(2 j+1)^{3}} X_{0}
$$

with $\lambda=2 \pi / k_{0}$. 
[1] R. C. Cross and M. S. Wheatland, "Modeling a falling slinky," American Journal of Physics, vol. 80, pp. 1051-1060, 2012.

[2] W. G. Unruh, "The falling Slinky," American Journal of Physics, vol. 80, pp. 1051-1060, 2012.

[3] R. J. Vanderbei, "The falling Slinky," The American mathematical Monthly, vol. 124, pp. 24-36, 2017.

[4] J. M. Bowen, "Slinky oscillations and the notion of effective mass," American Journal of Physics, vol. 50, pp. 1145-1148, 1982.

[5] R. A. Young, "Longitudinal standing waves on a vertically suspended slinky," American Journal of Physics, vol. 61, pp. 353-360, 1993.

[6] P. Gluck, "A project on soft springs and the slinky," Physics Education, vol. 46, pp. 178-185, 2010.

[7] G. Bradski, "The OpenCV Library," Dr. Dobb's Journal of Software Tools, 2000. 